\title{
19 RELATIONSHIP BETWEEN ALCOHOL MANGEMENT PLANS AND INJURY REDUCTIONS IN A REMOTE AUSTRALIAN COMMUNITY
}

doi:10.1136/injuryprev-2012-0405900.19

1,2Senserrick Teresa, 'yford Marilyn, ${ }^{1,2}$ Hinchcliff Reece, 'Boufous Soufiane,

${ }^{1,3}$ Clapham Kathleen, ${ }^{4}$ Torr Sally, Ivers Rebecca. ${ }^{1}$ George Institute for Global Health/ University of Sydney, 341 George Street, Sydney, Australia; ${ }^{2}$ University of New South Wales; ${ }^{3}$ University of Wollongong; ${ }^{4}$ Bourke District Hospital and Health Service

Background Alcohol management plans have been introduced in several remote communities in Australia to address a high rate of alcohol-related harm.

Aims/Objectives/Purpose To evaluate the impact on injury of the alcohol management plan in Bourke NSW (population 2175; 33\% Aboriginal) following introduction of takeaway alcohol restrictions in February 2009.

Methods Community and stakeholder interviews were conducted at $12(n=29)$ and 24 months $(n=19)$ post-restrictions. Hospital emergency presentations, admissions and police crime databases were analysed to compare injury at 12 months pre- and postrestrictions.

Results/Outcome Contested views were provided; however, the majority of interviewees reported visible and tangible benefits, such as reduced public drunkeness and violence. Police reported a $22 \%$ decrease in assaults. Hospital presentations $(\mathrm{N}=714)$ showed no change in the proportion involving injury (6\%) but a significant decline in the proportion of injury presentations involving alcohol (negative binomial regression $p=0.016$ ). Injury admissions were low $(\mathrm{N}=229)$, with few significant findings detected; however, head injuries decreased by 1.5 times.

Significance/Contribution to the Field Despite limitations of small numbers and alcohol status likely under-enumerated in hospital data, the results collectively indicate reductions in alcohol-related injury, supporting the increasing uptake of alcohol management plans. 